\title{
A Review on Congestion Management Methodologies and its Applications
}

\author{
Samar Abdulrahman Juma Al Raisi \\ Department of Chemical Engineering, \\ Caledonian College of Engineering, Seeb, Oman \\ samarabdulrahman1@gmail.com
}

\begin{abstract}
Since electric power industries are moving toward reconstruction of the power system, numerous challenges and key problems occur like congestion, operation, pricing, and management. In a power system, infringement of line loading and/or bus voltage limits because of different transactions during any time is called congestion. In the system, congestion might happen uneconomical operation and/or blackouts, a failure of the interconnected system and a disturbance. In the power system, Congestion Management plays a significant function in the operation of secure, economical, and stable operation in promising competitive electricity markets. This article surveys the conventional congestion management techniques for distribution networks using the meta-heuristic approaches in the current research literature. Moreover, it has to turn out to be a significant task in ever-developing challenges and factors that are forcing the development of novel methods. Also, this review discusses the congestion management techniques with diverse system applications are explained. In congestion management, the problems encountered with the roles and tasks of the methodologies are discussed. Several techniques and their compensation level are explained.
\end{abstract}

Keywords: Congestion Management; Meta-Heuristic Approaches; Optimal Power Flow; Applications; Challenges

\begin{tabular}{ll} 
Nomenclature & \\
\hline Abbreviations & Descriptions \\
\hline RTCM & Real-Time Congestion Management \\
FDR-PSO & Fitness Distance Ratio-PSO \\
ANN & Artificial Neural Network \\
FA-PSO & Fuzzy Adaptive-PSO \\
PSO & Particle Swarm Optimization \\
SVSI & Static Voltage Stability Index \\
CM & Congestion Management \\
CFA & Cat-Firefly Algorithm \\
MOPSO & Multi-Objective PSO \\
OPF & Optimal Power Flow \\
DRPs & Demand Response Programs \\
MO-GSO & Multi-Objective Glowworm Swarm Optimization \\
HNM-FAPSO & hybrid Nelder -Mead - Fuzzy Adaptive PSO \\
TLBO & Teaching-Learning-Based Optimization \\
MLCM & Multi-Line CM \\
FF & Firefly \\
APSF & Apparent Power Sensitivity Factor \\
DN & Distribution Networks \\
IP-PSO & Interior Point-Initialized PSO \\
DE & Differential Evolution \\
IPM & Interior Point Method \\
IGWO & Improved Grey Wolf Optimization \\
DMO & Distribution-level Market Operator \\
DS & Dynamic subsidy \\
DTO & Data Traffic Operator \\
GSA & Gravitational Search Algorithm \\
ALO & Ant Lion Optimizer \\
FAPSO & fuzzy adaptive PSO \\
&
\end{tabular}




\begin{tabular}{ll}
\hline S-PSO & standard PSO \\
OASIS & Open Access Same time Information System \\
APF & Active Power Flow \\
SVC & Static Var Compensators \\
OOP & Object-Oriented Programming \\
PTDF & Power Transfer Distribution Factors \\
NSGA-II & Nondominated Sorting Genetic Algorithm II \\
\hline
\end{tabular}

\section{Introduction}

The ability of transmission lines to broadcast electric power is controlled by numerous transfer limits like the thermal, voltage, and stability limit. The power system is referred to as congested while as a minimum any one of these parameters attains its utmost limit. ISOs encompass the main role to synchronize between the diverse market players and to control a reliable and secured power flow in a great deal loaded conventional transmission infrastructure [1].

An enhancement in power demand, unforeseen failures of generation, limitation on unscheduled power flow in lines, the structure of new lines, transmission lines tripping otherwise malfunctions of additional types of equipment are a few possible reasons for congestion. Considering this, reactive power sustains demand re-adjustment, generation rescheduling, et cetera are some choices which possibly will be developed to control the congestion issue [2].

In the power system, the novel features of congestion because of the physical and system restrictions of transmission systems. Moreover, the thermal constraint was considered as physical constraint while dynamic stability, nodal voltage limitation, reliability, and transient are referred to as system constraints that reason congestions in the transmission network [3]. It ought to be indifferent as rapidly as probable to make sure system stability and security of the system. If the outage happens often because of the congestion, the crisis turns out to be additional severe that affects the power quality and power systems equipment [4].

At present, the power industry deregulation has lead to the exhaustive procedure of transmission grids. In a restructured power market, the system is functioned close to its rated capability as every player in the market is demanding to increase to the extent that probable by using conventional resources [5]. In the transmission lines, congestion might happen because of the unforeseen contingencies like line outages, generation outages, and abrupt raise of load demand, types of equipment failure and the inadequacy coordination among transmission and generation utility. Generally, CM is considered a significant function done by SO to make the sure secured operation of the transmission system. Usually, there are 2 kinds of CM methods present in the state of the art that is cost-free techniques and non-cost free techniques; amongst them, subsequent technique mitigates congestion theoretically where the previous is associated with economics.

For CM, several techniques were recommended for power systems. These techniques comprise modification of load shedding; real power generation, and FACTS devices, is established to obtain frees of congestion of transmission lines. A method was presented to minimize both service costs and congestion by reducing real power loss using reactive power intensification [23]. A method to decrease the number of modifications of selected schedules and control methods to enhance CM. CM is based on the generation bids and associated congested line current flows. Using generation rescheduling CM exploiting generating company's block structures and FACTS devices were explained [22]. In a multi-machine system, CM by choosing the optimal site of TCSC exploiting the sensitivity indices based on system active and reactive power loss, real power flow, and ATC computation. Additionally, several soft computing techniques like PSO, GA, 3 NSGA-II, DE, SOS algorithm, and so on were presented for CM. The most important recently recommended optimization approach is the GWO algorithm.

The most important objective of this work is to present the review of the congestion management techniques and its applications. In literature, numerous approaches were developed by means of the contribution of congestion management, security enhancement and the effectiveness of the deregulated power market in precedent several decades.

\section{Literature Review}

\subsection{Meta-Heuristic Algorithm in Congestion Management}

In 2017, Mohammad Mahmoudian Esfahani et al [1], modeled a demand response program as well a real-time hybrid optimization approach was introduced in order to resolve the RTCM issue intended at 
discovering the optimal solution in a little period. Integrating an adaptive ANN besides using a modified PSO approach was developed as a real-time hybrid optimization algorithm. In 2019, Surender Reddy Salkuti and Seong- Cheol Kim [2], presented a new CM algorithm in an OPF model in the background of restructured power markets. The developed CM issue was devised by taking into consideration the 2 objective models. The developed multi-objective based on the CM issue was resolved by means of the MOGSO approach. In 2018, Sumit Verma et al [3] presented a TLBO approach for Congestion Management in a pool-based electricity market. The main aspire of using the TLBO approach was to competently ease congestion inline using minimum deviation at the initial generation and, thus, congestion cost. In 2019, S. Surender Reddy [4], worked on the CM issue, which was resolved via by exploiting the PSO, FA-PSO and FDR-PSO. Initially, generators were selected based on the sensitivity to the over-loaded transmission line, and afterward these generators were rescheduled to eliminate congestion in the transmission line. In 2017, Mohan Kashyap and Satish Kansal [5] proposed a hybridization of the FF approach and DE optimization search that controls the congestion efficiently through the rescheduling of generators fulfilling the system restraints both economically and technically in the deregulated market situation.In 2019, Saswati K Behera and Nalin K Mohanty [6], presented an IGWO approach to resolve CM issues. The enhancement in the GWO approach was done using a method that keeps a suitable balance amongst the exploration and exploitation stages of the approach and an enhanced formulation to decide Grey Wolves' locations during the course of the iterations. In 2016, Naraina Avudayappan and Deepa S N [7], worked on the multi-objective function shown to find OPF, and SVSI was resolved exploiting the developed optimization approach known CFA. Moreover, to revive the congestion in line multiple multi-type FACTS devices were optimally positioned by taking into consideration loading restricts of the transmission network. In 2018, Fariborz Zaeim-Kohan et al [8] worked on the MOPSO technique was used for transmission Congestion Management taking into consideration DRPs and generation rescheduling. Total operation/DR cost, emission and raising the transmission lines loading were the objective models of this issue. In 2016, J. Jeslin Drusila Nesamalar et al [9], developed an HNM-FAPSO for an MLCM issue, which was a nonlinear optimization issue in the deregulated power system. The generators that were sensitive to congested lines were chosen based on a new APSF and were rescheduled exploiting the HNM20 FAPSO algorithm. In 2017, Brijesh Singh et al [10], introduced a novel optimization algorithm, the IP- PSO algorithm was to resolve the CM issue. The algorithm straps up the advantages of both IPM and PSO algorithm. In 2017, P. Valsalal, S. Thangalakshmi [11], worked on the weak line, which was recognized exploiting the OPF performance index by means of the novel objective model by means of developed hybrid Fish - Bee approach. Moreover, the position of UPFC at a suitable line minimizes branch loading and decreases the voltage deviation. In 2018, Rajagopal Peesapati et al [15], proposed a hybrid algorithm consisting of several approaches such as GSA and FAPSO. The hybridization of the methods was performed to encompass integrated benefits such as global search ability and local search ability of GSA and S-PSO methods, correspondingly. Moreover, the fuzzy system based dynamic inertia weight was integrated into the S-PSO method to encompass precise nonlinear search space. In 2016, Sumit Verma and Vivekananda Mukherjee [17], worked on the intention of Congestion Management is to get rid of the congestion in lines whilst fulfilling all restraints about the least congestion cost. Here, a generation rescheduling-based method for CM in the electricity market exploiting a new ALO method was proposed.

\subsection{Other Approaches in Congestion Management}

In 2019, Athina Thanou et al [16], addressed the congestion issue in such a probabilistic and the unsure environment, price as an effectual method to drive visitor actions incompetent operation points, protecting museum operation stability. The equivalent issue to determine the time invested by each visitor at museum shows toward optimizing their attained knowledge, as stated using a correctly modeled prospect-theoretic utility functions using pricing, was devised and delighted as a noncooperative game. In 2018, Arash Asrari et al [12] developed a day-ahead market structure for CM in smart distribution networks. The developed model presents a stand for association among DMO and DTO to ease congested feeders hence data transmission traffic among market participants was efficiently controlled in a smart grid. In 2016, Shaojun Huang et al [13] developed a novel DS algorithm for CM in DNs, such as market technique, mathematical formula during a 2-level optimization, and technique to resolve optimization by reduction the linearization and constraints. In 2017, Despina I. Koukoula, and Nikos D. Hatziargyriou [14] developed a gossip method-based model to control power flows on radial distribution grids using decentralized demand management. Demand flexibility was related by means of a cost-like effectiveness function stating the problem reasoned by limitation. 


\section{Congestion Management Techniques}

Two main paradigms might be used for $\mathrm{CM}$ such as not-cost-free means and cost-free means. The previous comprise actions similar to the operation of transformer taps or failure of congested lines, phase shifters, else FACTS devices. These means are termed as, 'cost free' because the marginal costs (and not the capital costs) involved in their usage, are nominal. Non-cost free methods include re-dispatch of generation and curtailment of pool loads and/or by curtailment of bilateral networks. The former method relieves congestion technically, while the latter being related more with economics.

Between the aforesaid 2 main methods, cost free means do have benefits like not involving economical matters; so generation companies and distribution companies will not be involved. Therefore, optimal operation of FACTS devices as one such technology can minimize the transmission congestion as well as leads to superior usage of the conventional grid infrastructure, beside with many other advantages.

\subsection{Rescheduling Generation}

This guides to generation operation on an equilibrium point absent from one decided by equivalent gradual costs. In the dispatch framework, mathematical formulations of pricing tools might be integrated and the equivalent cost signals attained. These cost signals might be exploited for congestion pricing and indicators to the market participant to reorganize their power extractions/injections so that congestion is evaded.

\subsection{Prioritization and Limitation of Transactions/ Loads}

A parameter referred to as willingness-to-pay-to-avoid-limitation. This is an effectual instrument in setting transaction limitation schemes that might subsequently be integrated into the OPF structure.

\subsection{Transmission Grid CM}

In the electricity market, diverse techniques are exploited to systematically manage transmission congestion for diverse electricity market systems of diverse regions and countries. In [21], a study on CM at abroad and home was potted; close association among CM and market transaction scope, market trading mode, dynamic CM and congestion pricing was explained in diverse classification. Nevertheless, the CM occurred with the optimization, and network control parameters exploiting the FACTS device were not explained. Fig 1 demonstrates the transmission congestion management techniques.

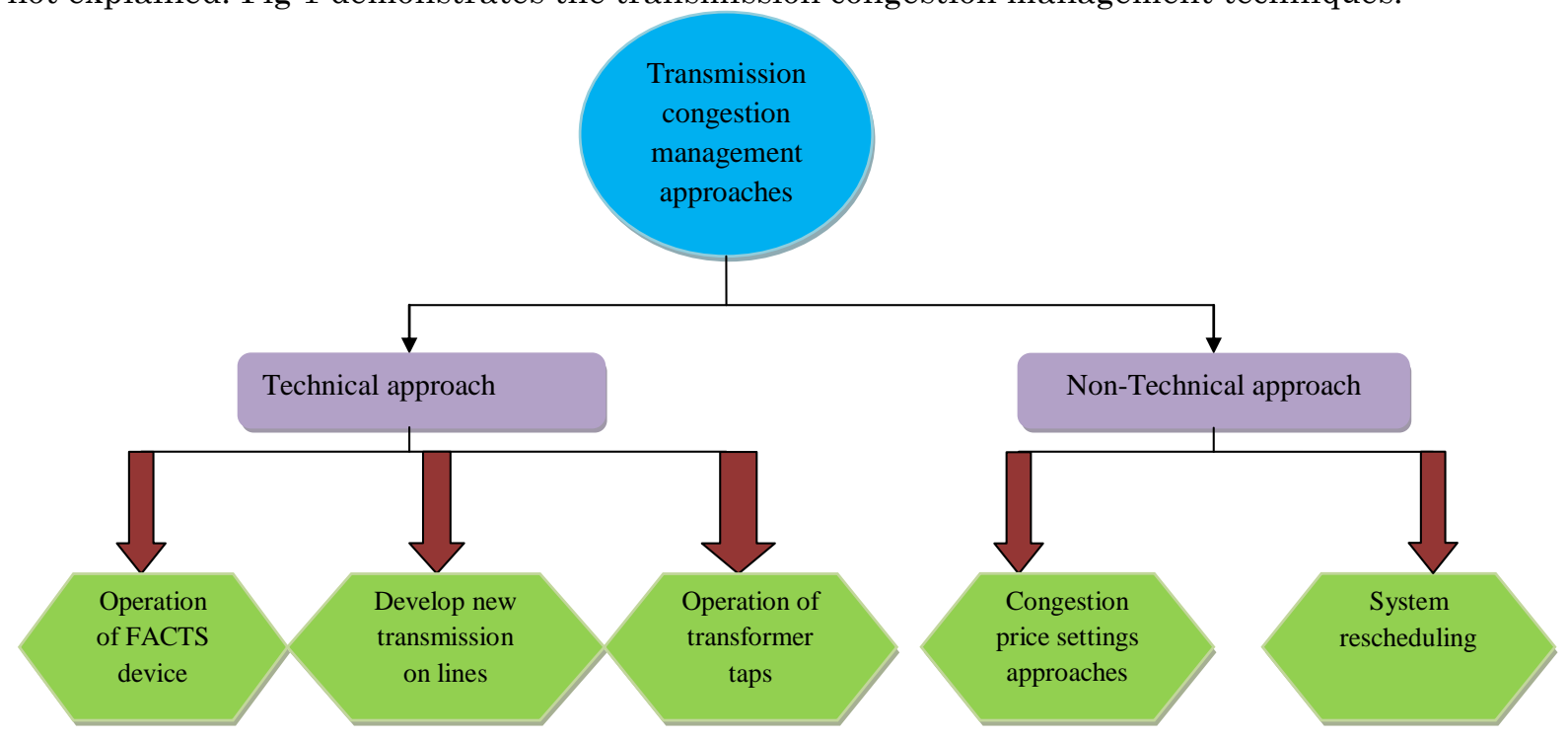

Fig. 1. Diagrammatic representation of transmission congestion management techniques

\subsubsection{Transmission CM technique based on the Technical Means}

The network physical characteristics must be occupied into contemplation initially while its methods to the behaviour of eradicating congestion since transmission congestion are occurred by inconsistency among the capacity of the network transmission and the transmission plan. From a long-term viewpoint, enhancing network capacity transmission is the main basic solution to the congestion issue. 


\subsubsection{Transmission CM technique on the basis of the Market Means}

Technical and physical techniques are restricted by factors like the performance of the equipment and capital investment, and geographic environment. Simultaneously, while the transmission system cannot convene required transmission plan, in circumstances of resource optimization and fair competition, by means of the reason of economy, numerous governments set up an opposition method and espouse price means to alter decrease otherwise augment of transaction volume, thus minimizing overload line power flow.

The CM technique on the basis of the electricity market economy was extensively exploited in international and domestic power systems. In the electricity market, several CM methods ought to face how to show market participants to alter their power generation injection and a power load. This is an important problem in the model of the power market method.

\subsection{Congestion Management Techniques}

In a deregulated power system, there are numerous techniques for CM. It is shortened consistent with the generation, transmission and the end-users side that are whatsoever the method exploited for CM while attempted from generation side and rapidly. Fig. 2 demonstrates the schematic diagram of congestion management.

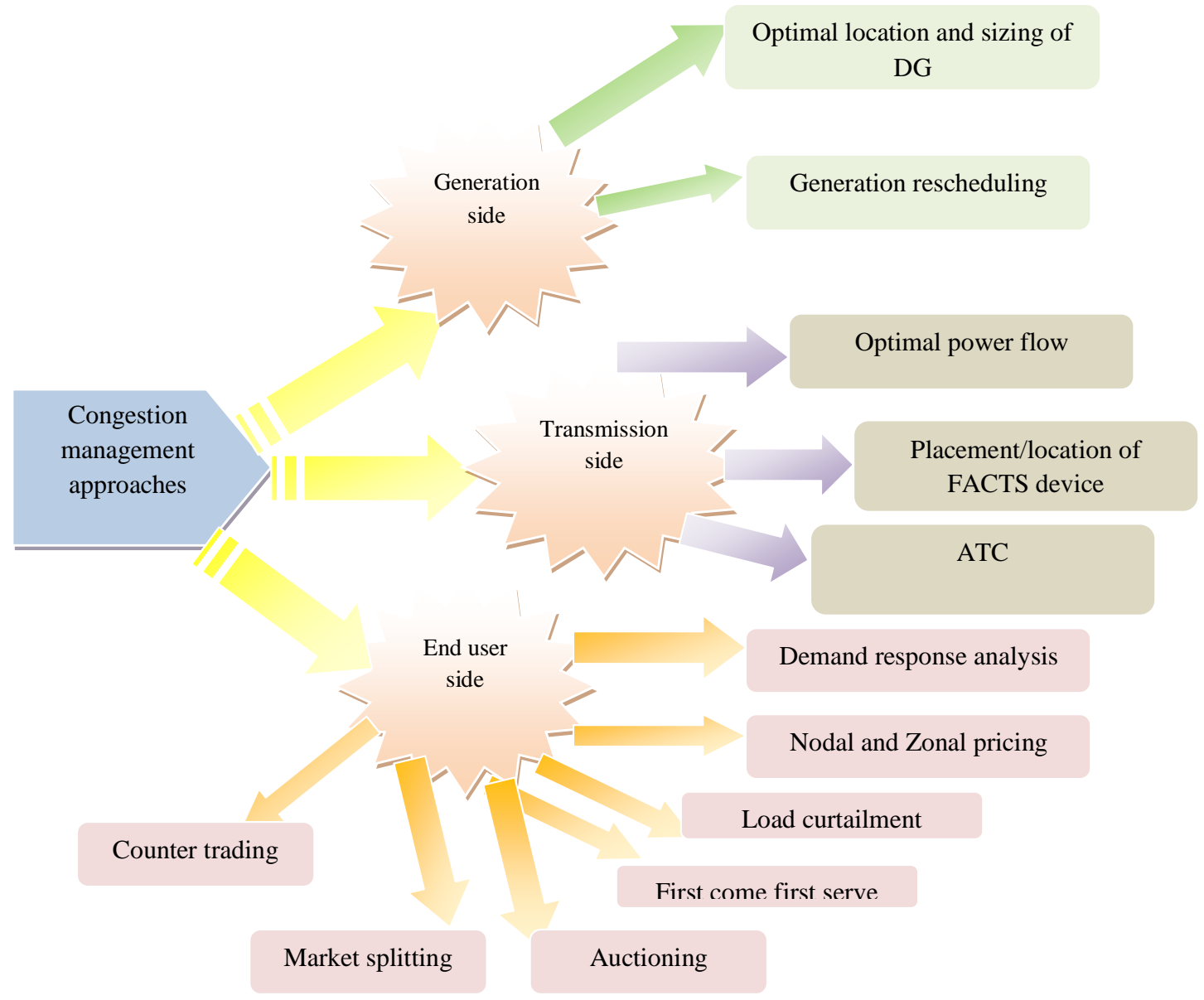

Fig. 2. Schematic diagram of congestion management methods in deregulated power system

$\mathrm{CM}$ can be generally categorized into 2 two systems. They are non- price-free measures and price-free measures. In the price-free measures, TSO would be setting the novel topology for transformer tabs with generic compensation devices for example phase modifiers and FACTS - Flexible AC Transmission System devices.

These are price-free devices that develop one-third of the total cost in their supposed utilize. The noncost-free measures comprise producing energy rescheduling and load limitation transactions. In loadshedding and generation rescheduling a method is developed for screening and ranking of contingencies. Diverse systems undertake congestion in dissimilar manners. 


\section{FACTS Devices Application in Congestion Management}

In the CM, the major limitation is the deregulation of power markets that is to supply power with economical and powerfully. Congestion in the line raises the cost of the supply unit beside the remaining congestion costs. The most important task of the FACTS devices is to raise the transfer capability of the transmission line. Also, decreases the congestion cost by carrying stability limit, voltage control, of the power system and reactive power recompense. A few FACTS devices such as SSSC, UPFC are recommended as optimal reactive power compensating devices. A closed-loop controller with DC-link and UPFC in amalgamation presents supporter pricing for the operating system in their imitations in demanding tasks.

\subsection{Congestion Management Price Control Theme}

In this topology, the system is categorized into multiple pricing units. The system using the extreme generating unit cost will encompass lower pricing and utilizing extreme power demand will have additional pricing. Here, the smudge market tenders should have to give separate bids for particular areas. In addition, it offers the network for area price computation and combines market CM.

\subsection{Congestion Management on ATC}

In the ATC the account of day to day and hour to hour is uploaded on the website known as OASIS. For assigning any transaction one has to OASIS web page to encompass report concerning this transaction details. OASIS proposes the computerized scheduling that as a prospect extent to ease for data sharing in the energy market in the direction of controlling congestion.

\subsection{Congestion Management on OPF}

The role of the OPF method is to minimize the generation pricing and present advantage to consumers regarding power system parameters. The OPF can be used efficiently while the system is congested aids in verifying overloading problems. An entire matrix for CM consists of bilateral markets, pool markets environment, and multilateral markets for power balance circumstances and limitation schemes. The fundamental object of limitation policies is to reduce deviations from scheduled transactions. In multilateral transactions, the system consistency is evaluated by CM which sequentially outcomes in minimized limitations and congestion charge reasonably the bilateral transactions.

An optimization resolution is called as decentralized risk-based CM that can be exploited to determine the thermal overloads. A soft approach called OPF control is exploited extensively in scheduling and minimizing congestion charges in the most of the congested system.

\section{Artificial Intelligence Approaches in Congestion Management}

The literature [18] developed a method of PSO as an algorithm exploited for the congestion management in power markets to reduce the generator rescheduling costs and alleviate congestion. IEEE 30-bus system was exploited and examined utilizing the exploit of PSO and at the end show that PSO is a better algorithm for CM. Both load line loading, and bus voltage security constraints, are economically handled with the PSO approach [18]. In deregulated power systems, the literature [19] exploited PSO approaches for controlling the congestion. The developed technique economically alleviates line overloads and it was evaluated with the DR method.

The most important apprehension in managing congestion is lines limit transport capability and available generation ability.

The study [20] developed a GA to resolve arithmetical formulation issues for system congestion. GA technique is exploited using the GR method or FACTS devices that functioned as an amalgamation of $\mathrm{CM}$ approaches. The developed approaches are obliging optimization tools capable to alleviate congestion and reduce system costs. Optimization exploiting a multi-objective algorithm, NSGA-II is exploited to resolve the optimization issue in a GR congestion technique. The developed CM technique with 2 objectives such as optimizing transmission line overload, and the congestion costs in the system, therefore, developed the NSGA-II optimization algorithm to be exploited.

The developed techniques efficiently alleviate the congestion inexpensively using the reduced shifts in the GR algorithm that shows that the NSGA-II algorithm is a competent algorithm needed while deals about more than one objective of CM. A fuzzy Logic optimization algorithm is presented using the purpose of control APF for CM by exploiting FACTS devices. The efficiency of the approach was examined effectively and the congestion is comforted with no rescheduling procedure in the system. The 
Fuzzy based technique was proposed to determine the optimal position of TCSC to manage APF and decrease of congestion in a transmission line. The developed technique aimed to structure a precedence list of optimal positions for TCSC shows the efficiency of the fuzzy optimization algorithm.

Since deregulated power markets experience from congestion, FACTS devices are exploited to reduce power flows in loaded lines to augment constancy and minimize losses of power. The fuzzy approach is presented to choose optimal positions for FACTS devices to control APF and alleviate congestion in the transmission line.

The optimization methods for the best choice, position, and the size of TCSC and SVC to decrease line losses, decrease congestion, and enhance voltage stability using the exploited of DE.

\section{Challenges and Issues in Congestion Management}

$\mathrm{CM}$ is a demanding chore for sustaining stability and system security. In demand-side management, the most important challenges are to deal with competence, uncontrolled loads, and climate change problems because of unsuitable market structure, need infrastructures and incentives. There are numerous issues and challenges in CM that are spotlighted as follows:

* Since it is renowned with the intention of the optimal manner to control the congestion is to reschedule active power however real power scheduling alters reactive power flows and might reason other issues of the power system. Hence, several periods, reactive powers besides using the active power rescheduling are presented that raises the difficulty of dispatch issue. At the restricted time, load shedding on the basis of the obtainable generation rescheduling and contracts can be done.

* In current smart grid proposals, ease of use of DGs sources can also be used on the other hand their size and appropriate position is significant. Congestion must be controlled rapidly to evade the security and stability issues, and consequently a rapid and dependable method, are required.

* Several existing and non-existing optimization approaches are used to resolve the CM issues however there are still appropriate computation intelligent approaches that are required that can be vigorous over system changes.

- Actually, the CM is a multi-objective complex issue and consequently its modeling is significant. There are various techniques developed in the state of the art exploiting few estimated approaches to resolve the issue rapidly other than they undergo from local optimal.

- Several sensitivity factors and PTDF are developed other than through the stressed circumstance, the accurateness of these approaches is the main apprehension. Precise modeling raises the computational burden and consequently, a high-quality tradeoff among estimated and precise modeling of the difficulty is required.

* There might have FACTS devices and its operation is exploited to alleviate congestion in systems. Nevertheless, its operation cost, and other financial problems, should be considered. The mechanism of these devices might mainly for enhancing the system constancy, security, and so on therefore the tradition for congestion can be represented as a less important objective.

- Numerous devices are exploited to manage congestion however control action cost might be an additional problem and consequently optimal control action a well as management has to be represented in the system.

* There are market-based CM approaches. The organization of transmission rights and the transmission network can be correctly used when controlling the congestion.

\section{Future Scope in Congestion Management}

$\mathrm{OPF}$ is progressively being exploited for transmission pricing and the transaction estimation in open access transmission systems. From the scenario studies performed in this study, it was evident that interconnection among market players is complex.

In this field, future work might concentrate on quantifying the economic risk that occurred by market players because of the differences in their inclination to pay to evade the limitation. Additionally, research might be performed on modeling the various dispatch and limitation schemes. The sensitivity technique to determine the optimal positions of FACTS devices can at optimal present an estimated design regarding the optimal position for those devices in a deregulated environment. More reliable techniques required to be adopted for this. Moreover, there is a requirement to use OOP techniques to the issue of OPF in a deregulated environment. That would ease the development of easier and reasonable OPF packages. 


\section{Conclusion}

Congestion network was a hazard to security, and the power industry economy in the power system. In deregulated power markets, congestion management has to turn out to be one of the important tasks for system operators to deal with congestion in the transmission network. In literature, numerous approaches were developed using aspire of congestion management, security enhancement and the effectiveness of the deregulated power market in precedent several decades. Moreover, this survey article details the diverse techniques/approaches of CM in precedent for several years and presents a complete outline of congestion management techniques. An inclusive challenge was done to exhibit the significance of $\mathrm{CM}$ technique to alleviate the congestion issue that was an up-and-coming development in numerous researches.

\section{References}

[1] Mohammad Mahmoudian Esfahani, Ahmed Sheikh, Osama Mohammed,"Adaptive real-time congestion management in smart power systems using a real-time hybrid optimization algorithm",Electric Power Systems Research, Volume 150, Pages 118-128, September 2017.

[2] Salkuti, Surender \& Kim, Seong-Cheol. (2019). Congestion Management Using Multi-Objective Glowworm Swarm Optimization Algorithm. Journal of Electrical Engineering \& Technology.

[3] Sumit Verma, Subhodip Saha, V. Mukherjee,"Optimal rescheduling of real power generation for congestion management using teaching-learning-based optimization algorithm", Journal of Electrical Systems and Information TechnologyVolume 5, Issue 3December 2018, Pages 889-907.

[4] S. Surender Reddy; S.A. Wajid,"Swarm intelligent-based congestion management using optimal rescheduling of generators",International Journal of Bio-Inspired Computation (IJBIC), Volume. 13, Num. 3, 2019.

[5] Mohan Kashyap \&Satish Kansal,"Hybrid approach for congestion management using optimal placement of distributed generator",International Journal of Ambient Energy,Volume 39, 2018 - Issue 2, page num-132$142,2016$.

[6] Saswati K Behera, Nalin K Mohanty,"Congestion management using thyristor controlled series compensator employing Improved Grey Wolf Optimization technique" The International Journal of Electrical Engineering \& Education, 8 Jan 2019.

[7] NARAINA AVUDAYAPPAN Deepa S N, "Congestion management in deregulated power system using hybrid Cat-Firefly algorithm with TCSC and SVC FACTS devices",COMPEL - The international journal for computation and mathematics in electrical and electronic engineering,Volume. 35 Iss 5, 2016.

[8] Fariborz Zaeim-Kohan, Hadi Razmi, Hasan Doagou-Mojarrad,"Multi-objective transmission congestion management considering demand response programs and generation rescheduling", Applied Soft Computing, Volume 70, page no. 169-181, Sept. 2018.

[9] J. Jeslin Drusila Nesamalar, P. Venkatesh, S. Charles Raja,"Managing multi-line power congestion by using Hybrid Nelder-Mead - Fuzzy Adaptive Particle Swarm Optimization (HNM-FAPSO)" Applied Soft ComputingVolume 43June 2016Pages 222-234.

[10] Brijesh Singh Ranjit Mahanty Shiv P. Singh,"Social welfare maximization for congestion management in multiutility market using improved PSO incorporating transmission loss cost allocation",Volume28, Issue9, September 2018.

[11] P. Valsalal, S. Thangalakshmi," Transmission Line Congestion Management Using Hybrid Fish-Bee Algorithm with Unified Power Flow Controller",January 2007.

[12] A. Asrari, M. Ansari, J. Khazaei and P. Fajri, "A Market Framework for Decentralized Congestion Management in Smart Distribution Grids Considering Collaboration Among Electric Vehicle Aggregators," IEEE Transactions on Smart Grid, vol. 11, no. 2, pp. 1147-1158, March 2020.

[13] S. Huang and Q. Wu, "Dynamic Subsidy Method for Congestion Management in Distribution Networks," IEEE Transactions on Smart Grid, volume. 9, number. 3, page no. 2140-2151, May 2018.

[14] D. I. Koukoula and N. D. Hatziargyriou, "Gossip Algorithms for Decentralized Congestion Management of Distribution Grids," in IEEE Transactions on Sustainable Energy, volume. 7, number. 3, page no. 1071-1080, July 2016.

[15] R. Peesapati, A. Yadav, V. K. Yadav and N. Kumar, "GSA-FAPSO-Based Generators Active Power Rescheduling for Transmission Congestion Management," in IEEE Systems Journal, volume. 13, number. 3, page no. 3266 3273, Sept. 2019

[16] A. Thanou, E. E. Tsiropoulou and S. Papavassiliou, "A Sociotechnical Approach to the Museum Congestion Management Problem," in IEEE Transactions on Computational Social Systems, vol. 7, no. 2, pp. 563-568, April 2020.

[17] S. Verma and V. Mukherjee, "Optimal real power rescheduling of generators for congestion management using a novel ant lion optimiser," in IET Generation, Transmission \& Distribution, volume. 10, number. 10, page no. 2548-2561, 772016.

[18] S. Chanda and A. De, "Application of Particle Swarm Optimization for relieving congestion in deregulated power system," IEEE Transactions, page no. 837-840, 2011.

[19] N. Kinhekar, N. P. Padhy, and H. O. Gupta, "Particle Swarm Optimization based Demand Response for residential consumers," IEEE Transactions, pp. 1-5, 2015. 
[20] S. M. H. Nabavi, A. Kazemi, and M. A. S. Masoum, "Congestion management using Genetic Algorithm in deregulated power environments," International Journal of Computer Applications, vol. 18(2), page no. 19-23, 2011.

[21] Zhang Y P, Jiao L W, Chen S S, et al. A survey of transmission congestion management in electricity markets[J], Power System Technology, 2003.

[22] Subramanyam T C,Tulasi Ram S S,Subrahmanyam J B V,"An Adaptive Chicken Swarm Algorithm to Solve Optimal Power Flow Problem Considering FACTS Device",Journal of Computational Mechanics, Power System and Control (JCMPS), Volume 2, Issue 1, January 2019.

[23] Harish Kumar V C,Amala Shanthi S,"Hybrid OBL-GO Algorithm to Support Damping Oscillations in Coordinated Control of UPFC and TCSC",Journal of Computational Mechanics, Power System and Control (JCMPS), Volume 3, Issue 1, January 2020. 